Research Paper

\title{
The Comparison Between Positive and Negative Symptoms Severity in Prolonged Methamphetamine-induced Psychotic Disorder and Schizophrenia
}

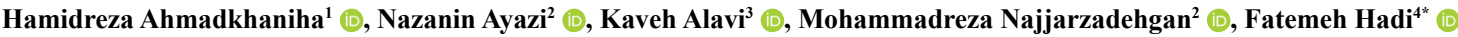 \\ 1. Research Center for Addiction and Risky Behaviors, Iran University of Medical Sciences, Tehran, Iran. \\ 2. Department of Psychiatry, School of Medicine, Iran University of Medical Sciences, Tehran, Iran. \\ 3. Department of Psychiatry, Mental Health Research Center, School of Behavioral Sciences \& Mental Health (Tehran Institute of Psychiatry), \\ Iran University of Medical Sciences, Tehran, Iran. \\ 4. School of Medicine, Shahid Beheshti University of Medical Sciences, Tehran, Iran.
}

\begin{tabular}{|c|c|}
\hline $\begin{array}{l}\text { Use your device toscan } \\
\text { and read the artice online }\end{array}$ & ditation Ahmadkhaniha, H. R., Ayazi, N., Alavi, K., Najjarzadehgan, M. R., \& Hadi, F. (2022) The Comparison \\
\hline 口p不葫回 & sitive and Negative Symptoms Severity in Prolonged Methamphetamine-Induced Psychotic Disorder and \\
\hline Fist & Schizophrenia. Basic and Clinical Neuroscience, 13(3), 325-334. http://dx.doi.org/10.32598/bcn.2021.2837.1 \\
\hline 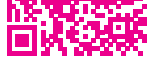 & http://dx.doi.org/10.32598/ben.2021.2837.1 \\
\hline
\end{tabular}

\section{(c) (1) (8)}

Article info:

Received: 22 Jul 2017

First Revision: 15 May 2020

Accepted: 10 Oct 2020

Available Online: 01 May 2022
Keywords:

Methamphetamine, Schizophrenia, Psychotic disorder, Positive and negative symptoms severity, Brief Psychiatric Rating Scale, Prolonged methamphetamineinduced psychosis

\section{AB S T RAC T}

Introduction: This study aims to compare the positive and negative symptoms of schizophrenia in patients who had psychotic symptoms more than one month after discontinuation of methamphetamine abuse. These factors were analyzed by the positive and negative syndrome scale (PANSS) questionnaire.

Methods: Sixty participants were selected from patients referred to Iran Psychiatric Hospital with psychotic symptoms (delusions or hallucinations, disorganized behavior, and speech). The control group was 30 patients with schizophrenia based on a semi-structured interview according to DSMIV-TR (SCID). Thirty patients with a prolonged methamphetamine-induced psychotic disorder were also placed in the case group. For both groups of patients, questionnaires of PANSS, Brief Psychiatric Rating Scale (BPRS), and Global Assessment Of Functioning (GAF) were filled out after obtaining the companions' consent. The scale scores were compared between groups. We used the Mann-Whitney and the Chi-square test to evaluate the mean values of PANSS, BPRS, and GAF scores between the two groups.

Results: here was an insignificant difference in positive and general pathology scores between the two groups, but the total score of negative symptoms in the schizophrenia group was significantly higher than in the group of prolonged methamphetamine psychotic disorders $(\mathrm{P}=0.034)$. Average scores of uncooperativeness (0.008), difficulty in abstract thinking (0.004), motor retardation (0.002), unusual thought content $(0.001)$, and hostility $(0.011)$ in the schizophrenia group were significantly higher than those in the prolonged methamphetamine psychosis.

Conclusion: The results showed that most of the disturbances in patients with schizophrenia might be more influenced by the expression of cognitive disabilities than those with methamphetamine psychosis. The difference in negative symptom scores suggests that schizophrenia and prolonged methamphetamine psychotic disorder can be two different disorders.

\footnotetext{
* Corresponding Author:
}

Fatemeh Hadi, MD.

Address: School of Medicine, Shahid Beheshti University of Medical Sciences, Tehran, Iran.

Tel: +98 (21) 22439770

E-mail: fatemehadi88@yahoo.com 


\section{Highlights}

- General and positive symptoms scores don’t have significant differences.

- Negative symptoms are much more in schizophrenia.

- Uncooperativeness, unusual thought content and, motor retardation have more scores in schizophrenia.

\section{Plain Language Summary}

In clinical practice, Schizophrenia and prolonged methamphetamine-induced psychotic disorder have some similar mental presentations. Additionally, in scientific literature, there is scarce evidence about these similarities. In this regard, this research was designed to investigate the aforementioned obscurity. Determination of similarities and differences between them helps us to address these disorders in terms of treatment and follow-up and awareness of their prognosis of them. This research is a case-control study in which we examine positive and negative psychotic symptoms in schizophrenia and prolonged methamphetamine-induced psychotic disorder. Researchers investigated psychotic symptoms with positive and negative syndrome scale (PANSS), brief psychiatric rating scale (BPRS), and global assessment of functioning (GAF) questionnaires. Moreover, results demonstrate general and positive symptoms scores don't have many differences but negative symptoms are much more in patients with schizophrenia than in patients with a prolonged methamphetamine-induced psychotic disorder. Also, other features like uncooperativeness, unusual thought content, motor retardation, difficulty in abstract thinking, and hostility have higher scores in schizophrenia than the others. In conclusion, this research showed that these disorders are two distinct disorders with some similarities in positive symptoms but not in all features. So, some studies can be designed about why there are similarities between them?

\section{Introduction}

he pattern of drug abuse in Iran in recent $\mathbf{T}$ years has undergone a lot of changes from traditional opiates (like opium) to modern ones (like heroin) and synthetic materials (such as methamphetamine) (Samii, 2003). In the wake of the change in substance abuse, complications and consequences associated with them have also hung, and treatment centers are now facing various disorders of the emerging materials as well. One of the most significant symptoms is a psychotic disorder due to the use of methamphetamine, which has occupied a significant proportion of psychiatric hospital beds for patients with this disorder (Zarghami, 2011).

Methamphetamine enters the central nervous system and causes sudden dopamine release in the brain that stimulates neurons and increases the feeling of pleasure and physical movements. After a long period of use, symptoms of movement disorders like Parkinson disease and depression appear in patients. Furthermore, from the onset, methamphetamine leads to irreversible damage to the brain, some complications of which include psychosis, memory loss, agitation, aggres- sion, disorganized behavior, and brain tissue damage (Zarghami, 2011).

One of the psychological effects of methamphetamine abuse is creating mental illnesses like schizophrenia, including visual and auditory hallucinations, persecutory delusion, and aggression(Samii, 2003). Psychosis due to methamphetamine use leads to severe symptoms often associated with subsequent hospitalization (Zarghami, 2011).

There are several hypotheses regarding the positive and negative symptoms of stimulant abuse, causing dopamine reuptake inhibition by interfering with the function of the dopamine transporter (DAT) and increasing dopamine concentration in the synaptic cleft. Either methamphetamine or amphetamine is directly involved in the reward system, and due to the release of dopamine from the dopaminergic neurons and the norepinephrine in the synaptic cleft of the nucleus accumbens neurons, they cause euphoria and addiction (Vidal-Infer, RogerSánchez, Daza-Losada, Aguilar, Miñarro, \& RodríguezArias, 2012). The same effects of dopaminergic neurons can lead to methamphetamine-induced psychosis (Lohr et al., 2015; Shin et al., 2012). 
On the other hand, different hypotheses have been proposed that after using methamphetamine, some people will develop prolonged psychosis that lasts for more than one to several months and even persistent psychosis (Chen et al., 2005). A hypothesis states that patients with schizophrenia tend to abuse drugs due to their underlying psychiatric disorder. There is evidence that patients with underlying psychological disorders are increasingly vulnerable to mandatory use of stimulants such as methamphetamine. This issue has been raised, especially for patients with schizophrenia (Li, Lu, Xiao, Li, He \& Mei, 2014). Much evidence is mounting for this comorbidity, but compelling evidence from animal studies found that this condition may be due to common vulnerabilities and the creation of psychosis in schizophrenia and stimulants-induced psychotic disorder (Ikeda et al., 2013). The relationship between methamphetamine abuse and the development of psychosis has been highly discussed in recent years, but many questions about this complex relationship have remained unanswered.

One of these questions is whether the prolonged methamphetamine-induced psychotic disorder is the same as schizophrenia, triggered by methamphetamine, or is a different disorder clinically similar to schizophrenia. In this study, we compared the clinical symptoms and investigated the clinical similarities between the two disorders.

\section{Materials and Methods}

In this study, we aimed to compare the positive and negative symptoms of schizophrenia with prolonged psychosis due to methamphetamine abuse which lasted over the past six months and was obtained through factor analysis testing on data from the positive and negative symptoms severity (PANSS) questionnaire.

This cross-sectional study was conducted from April 2013 for 12 months in Iran Psychiatry Hospital in Tehran, Iran. The patients were selected amongst those referred to Iran Psychiatric Hospital with psychotic symptoms (delusions, hallucinations, disorganized behavior, and speech). The purposive sampling method was used to include only methamphetamine-induced psychotic and patients with first-episode schizophrenia. The patients of the case group had a diagnosis of prolonged methamphetamine psychotic disorder, with the duration of their psychotic symptoms taking more than one month but less than 6 months without repeating the usage of methamphetamine or psychogenic substances. The control group was patients di- agnosed with schizophrenia based on semi-structured interviews according to DSM-IV-TR (SCID). Whether patients needed to be admitted or not and after taking informed consent, they were included in the study. The study excluded patients with schizophrenia who were re-affected by psychotic symptoms due to methamphetamine use. Also, patients with polysubstance abuse, especially other stimulants like cannabis or ecstasy, and with a diagnosis of other psychotic disorders but schizophrenia were excluded from the study.

The patients with the first episode of schizophrenia were chosen because of both positive and negative symptoms; on the other hand, patients with chronic schizophrenia may be more influenced by medications and complications of the disorder. Patients with schizophrenia aged 18 to 50 were included and compared to patients in the same age group with prolonged methamphetamine psychotic disorders. Patients in both groups should not have positive urine tested for methamphetamine and amphetamine. Each participant received a questionnaire, a consent form to retrieve their demographic data, and an information sheet explaining the confidentiality of the participants' information. The completed questionnaires and signed consent forms were returned within one week and included their ID and a phone number so that they could be followed up in the case of missing data and matching data with participants' medical records. After data collection was finished, the data were processed anonymously. A structured clinical interview based on DSM-IV-TR symptoms of the patients was performed. Then the patients were explained during a short meeting to conduct questionnaires on the Positive And Negative Symptoms Scale (PANSS) (Kay, Fiszbein, \& Opler, 1987), brief psychiatric rating scale (BPRS) (Overall \& Gorham, 1962), and global assessment of functioning (GAF)(Hall, 1995), after giving their written consent.

Data collecting instrument characteristics and methodology

This study collected positive and negative symptoms data through PANSS, BPRS, and GAF questionnaires. Using records information and diagnosis examination were based on SCID (according to DSM-IV-TR). A psychiatry assistant determined inclusion criteria, gathered information, and completed the questionnaires. 
Table 1. Comparing demographic characteristics between the two groups of patients

\begin{tabular}{|c|c|c|c|c|}
\hline \multirow{2}{*}{\multicolumn{2}{|c|}{ Variables }} & \multicolumn{2}{|r|}{ No. (\%) } & \multirow{2}{*}{$\begin{array}{c}\text { P } \\
\text { (Chi-square Test) }\end{array}$} \\
\hline & & Schizophrenia & Methamphetamine Psychosis & \\
\hline \multirow{3}{*}{ Gender } & Male & $17(56.7)$ & $26(86.7)$ & \multirow{3}{*}{0.020} \\
\hline & & & & \\
\hline & Female & $13(43.3)$ & $4(13.3)$ & \\
\hline \multirow{2}{*}{$\begin{array}{l}\text { Education } \\
\text { level }\end{array}$} & Under diploma & $24(80)$ & $15(50)$ & \multirow{2}{*}{0.029} \\
\hline & Diploma \& higher & $6(20)$ & $15(50)$ & \\
\hline \multirow{3}{*}{ Employment } & Unemployed & $15(50)$ & $4(13.3)$ & \multirow{3}{*}{0.006} \\
\hline & Employed with a permanent job & $13(43.3)$ & 19(63.3) & \\
\hline & Employed without a permanent job & $2(6.7)$ & $17(56.7)$ & \\
\hline
\end{tabular}

\section{Data analysis}

The obtained data were analyzed by SPSS v. 22 statistical software. To describe the qualitative variables, we used prevalence and frequency. To evaluate the mean of PANSS, BPRS, and the GAF scores between the two groups, we used the Mann-Whitney test and, for qualitative variables, the Chi-square test.

\section{Ethical Considerations}

This study has approval from the Ethics Committee of Iran University of Medical Sciences with the ethical number 24153. All provisions of the Helsinki Declaration and Code of Ethics adopted by the Iran Ministry of Health were considered in this study.

\section{Results}

In each group, 30 patients with schizophrenia and prolonged methamphetamine-induced psychotic disorder were investigated. The Mean $\pm \mathrm{SD}$ age in the group with prolonged methamphetamine psychotic disorder was $33.2 \pm 6.9$ years, and in schizophrenia was $25.2 \pm 3.4$ years. The mean age was significantly lower in the schizophrenia group (Independent $t$ test: $\mathrm{P}=1.019, \mathrm{t}=0.036$ ).

As seen in Table 1, the frequency of male patients was higher in the prolonged methamphetamine-induced psychotic disorder group, and the group of schizophrenia patients was less educated and employed. The result from comparing demographic variables between patients with first-episode schizophrenia and prolonged methamphetamine-induced psychotic disorder is presented in Table 1 .

Table 2. Comparing negative symptoms based on positive and negative syndrome scale (PANSS) questionnaire between the two groups of patients

\begin{tabular}{|c|c|c|c|}
\hline \multirow{2}{*}{ Variables } & \multicolumn{2}{|c|}{ Mean \pm SD } & \multirow{2}{*}{$\begin{array}{c}\text { P } \\
\text { (Mann-Whitney Test) }\end{array}$} \\
\hline & Schizophrenia & Methamphetamine Psychosis & \\
\hline Emotional withdrawal & $2.73 \pm 0.22$ & $2.4 \pm 0.22$ & 0.201 \\
\hline Blunted affect & $2.5 \pm 0.23$ & $2.13 \pm 0.26$ & 0.153 \\
\hline Poor rapport & $2.73 \pm 0.19$ & $2.33 \pm 0.23$ & 0.473 \\
\hline Passive/apathetic social withdrawal & $2.67 \pm 0.18$ & $2.5 \pm 0.21$ & 0.069 \\
\hline Difficulty in abstract thinking & $3.066 \pm 0.18$ & $2 \pm 0.21$ & 0.004 \\
\hline Total negative symptoms score & $13.6 \pm 4.15$ & $11.77 \pm 5.1$ & 0.034 \\
\hline
\end{tabular}


Table 3. Comparing general psychopathology based on the positive and negative syndrome scale (PANSS) scale between the two groups of patients

\begin{tabular}{cccc}
\hline \multirow{2}{*}{ Variables } & \multicolumn{2}{c}{ Mean \pm SD } & P \\
\cline { 2 - 4 } & Schizophrenia & Methamphetamine Psychosis & 0.290 \\
\hline Active social avoidance & $2.73 \pm 0.18$ & $2.4 \pm 0.21$ & 0.278 \\
\hline Disorientation & $1.83 \pm 0.14$ & $1.50 \pm 0.12$ & 0.270 \\
\hline Poor attention & $2.31 \pm 0.23$ & $2.11 \pm 0.22$ & 0.417 \\
\hline Mannerisms and posturing & $2.9 \pm 0.25$ & $2.67 \pm 0.25$ & 0.394 \\
\hline Poor impulse control & $2.5 \pm 0.2$ & $2.27 \pm 0.35$ & 0.529 \\
\hline Anxiety & $2 \pm 0.25$ & $2.27 \pm 0.34$ & 0.528 \\
\hline Tension & $2.1 \pm 0.26$ & $2.43 \pm 0.34$ & 0.365 \\
\hline Depression & $2.07 \pm 0.23$ & $1.77 \pm 0.23$ & 0.173 \\
\hline Guilt feelings & $2.03 \pm 0.21$ & $1.63 \pm 0.24$ & 0.903 \\
\hline Somatic concern & $2.47 \pm 0.2$ & $2.53 \pm 0.26$ & 0.021 \\
\hline Disturbance of volition & $3.37 \pm 0.17$ & $2.67 \pm 0.22$ & 0.695 \\
\hline Total general psychopathology score & $26.83 \pm 6.23$ & $27.18 \pm 7.18$ & \\
\hline
\end{tabular}

NEUR:SCIENCE

The results of comparing the negative symptoms scale between the two groups of patients are presented in Table 2. Mean \pm SD PANSS total scores were $59.9 \pm 12.5$ in the schizophrenia group and $57.5 \pm 11.8$ in the prolonged methamphetamine group ( $\mathrm{t}$ test for independent samples: $\mathrm{t}=1.085 ; \mathrm{P}=0.282$ ). The subscale of difficulty in abstract thinking in the schizophrenia group was $3.066 \pm 0.18$, and in the methamphetamine group, it was $2 \pm 0.21$, which was significantly higher in patients with schizophrenia $(\mathrm{P}=0.004)$ (Table 2).

Also, the mean scores of conceptual disorganization, hostility, and disturbance of volition were significantly higher in the schizophrenia group (Table 3).

Table 4. Comparing the positive symptoms based on the positive and negative syndrome scale (PANSS) questionnaire between the two groups of patients

\begin{tabular}{cccc}
\hline \multirow{2}{*}{ Variables } & \multicolumn{2}{c}{ Mean \pm SD } & $\begin{array}{c}\text { P } \\
\text { (Mann-Whitney Test) }\end{array}$ \\
\cline { 2 - 4 } & Schizophrenia & Methamphetamine Psychosis & 0.003 \\
\hline Conceptual disorganization & $3.33 \pm 0.31$ & $2.01 \pm 0.22$ & 0.513 \\
\hline Delusions & $3.6 \pm 0.28$ & $3.37 \pm 0.29$ & 0.957 \\
Hallucinations & $3 \pm 0.27$ & $2.67 \pm 0.3$ & 0.274 \\
Hyperactivity & $2.17 \pm 0.27$ & $2.27 \pm 0.33$ & 0.011 \\
\hline Hostility & $3.57 \pm 0.13$ & $4.07 \pm 0.13$ & 0.852 \\
\hline Total positive score & $15.69 \pm 3.25$ & $14.4 \pm 4.27$ & 0.282 \\
\hline PANSS total score & $59.9 \pm 12.5$ & $57.5 \pm 11.8$ & Ne \\
\hline
\end{tabular}


Table 5. Comparing brief psychiatric rating scale (BPRS), and global assessment of functioning (GAF) between the two groups of patients

\begin{tabular}{|c|c|c|c|}
\hline \multirow{2}{*}{ Variables } & \multicolumn{2}{|c|}{ Mean \pm SD } & \multirow{2}{*}{$\begin{array}{c}\text { P } \\
\text { (Statistical Analysis) }\end{array}$} \\
\hline & Schizophrenia & Methamphetamine Psychosis & \\
\hline Anxiety & $2.27 \pm 0.2$ & $2.9 \pm 0.28$ & 0.908 \\
\hline Emotional withdrawal & $2.73 \pm 0.22$ & $2.3 \pm 0.21$ & 0.123 \\
\hline Conceptual Disorganization & $2.63 \pm 0.23$ & $2.83 \pm 0.26$ & 0.533 \\
\hline Guilt Feeling & $2.03 \pm 0.16$ & $2.27 \pm 0.19$ & 0.157 \\
\hline Tension & $3.2 \pm 0.21$ & $3.43 \pm 0.3$ & 0.529 \\
\hline Mannerism \& Posturing & $2.6 \pm 0.15$ & $2.53 \pm 0.23$ & 0.811 \\
\hline Grandiosity & $3.17 \pm 0.14$ & $3.13 \pm 0.25$ & 0.909 \\
\hline Depressed mood & $3.03 \pm 0.21$ & $2.87 \pm 0.27$ & 0.629 \\
\hline Hostility & $3.43 \pm 0.14$ & $3.33 \pm 0.2$ & 0.684 \\
\hline Suspiciousness & $2.73 \pm 0.16$ & $2.50 \pm 0.21$ & 0.391 \\
\hline Hallucinatory behavior & $1.45 \pm 0.26$ & $1.44 \pm 0.26$ & 0.376 \\
\hline Motor retardation & $3.70 \pm 0.22$ & $2.5 \pm 0.26$ & 0.002 \\
\hline Uncooperativeness & $3.27 \pm 0.13$ & $2.4 \pm 0.23$ & 0.008 \\
\hline Unusual thought content & $4.17 \pm 0.15$ & $3.3 \pm 0.2$ & 0.001 \\
\hline Blunted affect & $3.07 \pm 0.16$ & $3.07 \pm 0.16$ & 0.961 \\
\hline Excitement & $2.97 \pm 0.16$ & $2.47 \pm 0.19$ & 0.056 \\
\hline Disorientation & $2 \pm 0.16$ & $2.13 \pm 0.18$ & 0.586 \\
\hline Somatic concerns & $2.1 \pm 0.16$ & $2.13 \pm 0.19$ & 0.894 \\
\hline GAF & $23.14 \pm 3.51$ & $24.56 \pm 4.56$ & 0.739 \\
\hline BPRS total score & $54.06 \pm 10.01$ & $49.4 \pm 10.1$ & 0.060 \\
\hline
\end{tabular}

The Mean \pm SD score of BPRS in the schizophrenia group was $54.1 \pm 1$, and in the methamphetamine group, it was $49.4 \pm 10.1$ (independent sample t test: $\mathrm{t}=1.802 ; \mathrm{P}=0.077)$. The subscales of motor retardation, uncooperativeness, and unusual thought content were significantly higher in schizophrenic patients than in the other group (Table 5).

The Mean \pm SD GAF score in the group of schizophrenia was $23.14 \pm 3.51$, and in the methamphetamine group, it was $24.56 \pm 4.56$. The Mean $\pm \mathrm{SD}$ total positive symptoms score in the schizophrenia group was $15.69 \pm 3.25$, and in the methamphetamine group, it was $14.40 \pm 4.27$ (Table 4). BPRS and GAF questionnaire results of the comparison between the two groups of patients are shown in Table 5.

The total negative symptoms in schizophrenia score were significantly higher than in the methamphetamine group $(\mathrm{P}=0.034)$ (Table 2$)$. The total Mean $\pm \mathrm{SD}$ psychopathology score in the schizophrenia group was $26.83 \pm 6.23$, and in the methamphetamine group, it was $27.18 \pm 7.18$ (Table 3).

\section{Discussion}

Our study showed that patients with prolonged methamphetamine-induced psychotic disorder had in- 
significant differences in the total scores of positive symptoms and general psychopathology with schizophrenic patients. However, the total score of negative symptoms was significantly higher in the patients with the first episode of schizophrenia.

Many studies detected similarities in positive symptoms in patients with schizophrenia and patients with methamphetamine psychosis (Hajebi, Amini, Kashani, \& Sharifi, 2018; Medhus, Mordal, Holm, Mørland, \& Bramness, 2013; Srisurapanont, Arunpongpaisal, Wada, Marsden, Ali, \& Kongsakon, 2011). Moreover, in some studies, there were no significant differences between methamphetamine psychosis and schizophrenia using the BPRS or the PANSS (Hides et al., 2015; Okada et al., 2016). But Srisurapanont et al. suggested no difference between methamphetamine psychosis and schizophrenia on measures of psychomotor retardation, flattened affect, and poverty of speech which some of these are negative symptoms (Srisurapanont et al., 2011)

Our results showed that negative symptoms in schizophrenia patients were more severe because the etiology of schizophrenia and prolonged methamphetamine-induced psychotic disorder may be different.

Furthermore, the difference in negative symptoms is evident in studies similar to our study (Ali et al., 2010; Srisurapanont, Ali, Marsden, Sunga, Wada, \& Monteiro, 2003). For example, Hajebi et al. found that the severity of negative symptoms is different between the two groups (Hajebi et al., 2018).

Our results showed that the subscale of negative symptoms, including disturbance of volition, and impaired abstract concept, differed in the two groups. Also, the difference in the scale of general psychopathology, including motor retardation, unusual thought content, and non-cooperativeness and positive symptoms subscale, including hostility in schizophrenic patients, were significantly higher than the prolonged methamphetamine-induced psychotic patients.

The difference in unusual thought content could suggest that the form and content of thought in schizophrenia patients are affected more than the patients with methamphetamine-induced psychosis. Furthermore, in terms of their psychomotor, people with schizophrenia are slower than people with psychosis generated by methamphetamine use. The differences between criteria of violence and non-cooperation can be non-specific and are changing according to the proceeding with the condition of the interview and the interviewer. How- ever, disturbances in patients with schizophrenia may indicate further affecting their cognitive abilities compared to methamphetamine-induced psychosis patients.

In 2011, Eghtedari et al. investigated cognitive functions in patients with methamphetamine-induced psychosis and compared them with the control group; the results showed that methamphetamine-induced psychotic patients had significantly more functional defects in areas such as attention, working memory, and executive functions compared to the control group. Methamphetamine-induced psychotic patients showed higher and prolonged deficits in their frontostriatal systems, particularly the prefrontal cortex and neurological functions (attention, executive memory, and executive functions) (Eghtedari, Shariat, \& Farahani, 2012). On the other hand, studies have shown that schizophrenia, unlike methamphetamine-induced psychotic disorder, is associated with progressive and irreversible cognitive deficits in patients (Ezzatpanah, Shariat, \& Tehrani-Doost, 2014). Perhaps it can explain the difference in thought disorders in two groups of our patients in this way.

The results of our study showed that the mean GAF score was not significantly different between the two groups because the study samples were selected from the patients who had been admitted to a referral hospital whose severe dysfunction is leading to hospitalization.

On the other hand, investigating the symptoms in patients with prolonged methamphetamine psychosis can be a way to understand the etiology of schizophrenia better. According to the studies, the etiology of schizophrenia is still unclear, although proposed models have identified genetic markers and environmental pathogens as significant risk factors.

In our study, we did not investigate risk factors for persistent psychotic symptoms in patients with methamphetamine abuse; however, in a 10-year cohort study in California, to find an association between the drugs and schizophrenia, a group of inpatients with a diagnosis of substance use disorders were evaluated. According to this study, compared with other material abuse (other than cannabis), patients with methamphetamine-related disorders had a higher risk of developing schizophrenia (Callaghan et al., 2012). In another study, results support common predisposing factors for prolonged methamphetamine psychosis and schizophrenia (Ahmadkhaniha, Alavi, \& Hadi, 2019). 
Based on the study by Ujike $\mathrm{H}$ et al., in patients with recurrent psychosis, increased vulnerability to methamphetamine abuse is seen, and in case of recurrence abuse, this vulnerability will last a very long time. These features are called the sensitivity of methamphetamine abuse and could explain the recurrence of methamphetamine psychosis with stress and prolonged and intractable psychosis induced by substance abuse (Ujike \& Sato, 2004).

Researches show similarities and differences between methamphetamine psychosis and schizophrenia (Wearne \& Cornish, 2018). There are predisposing genes for schizophrenia spectrum disorders and psychosis induced by methamphetamine (Ahmadkhaniha et al., 2019). The threshold of the emergence of psychotic symptoms is lower in people who carry these genes, and their clinical conditions are probably less favorable. Thus, there is a complex relationship between methamphetamine use and psychosis where enough information is not available yet (Akiyama, Saito, \& Shimoda, 2011).

\section{Conclusion}

Results from the current study showed significant differences in negative symptoms profile and some general psychopathologies indicating differences in presentation of the two disorders but also similarities. So, these two disorders can be considered two separate conditions, and future studies should be designed to investigate these differences more (especially in etiology, clinical presentation, course of the disorder, and response to treatment).

The small sample size and significant differences in demographic factors between the two groups are important limitations. Also, there was a lack of simultaneous evaluation of risk factors of prolonged psychosis. Finally, we did not follow up with the patients and monitor their condition for a given period.

\section{Ethical Considerations}

\section{Compliance with ethical guidelines}

All ethical principles are considered in this article. The participants were informed of the purpose of the research and its implementation stages. They were also assured about the confidentiality of their information and were free to leave the study whenever they wanted, and if desired, the research results would be available to them. A written consent has been obtained from the subjects. principles of the Helsinki Convention was also observed. This study was approved by the Ethics Committee of the Iran University of Medical Sciences (Code: 24153).

Funding

This article is the thesis of Nazanin Ayazi supervised by Hamidreza Ahmadkhaniha at Iran Iran University of Medical Sciences.

\section{Authors' contributions}

All authors equally contributed to preparing this article.

\section{Conflict of interest}

The authors declared no conflict of interest.

\section{Acknowledgments}

We appreciate all of the personnel of Iran Psychiatry Hospital.

\section{Refrences}

Ahmadkhaniha, H. R., Alavi, K., \& Hadi, F. (2019). Prolonged methamphetamine-induced psychosis: Difference with schizophrenia. Acta Medica Iranica., 57(4), 235-243. [DOI:10.18502/acta.v57i4.1843]

Akiyama, K., Saito, A., \& Shimoda, K. (2011). Chronic methamphetamine psychosis after long-term abstinence in Japanese incarcerated patients. The American Journal on Addictions, 20(3), 240-249. [DOI:10.1111/j.1521-0391.2011.00124.x] [PMID]

Ali, R., Marsden, J., Srisurapanont, M., Sunga, A., Baigent, M., \& Monteiro, M. (2010). Methamphetamine psychosis in Australia, Philippines, and Thailand: Recommendations for acute care and clinical inpatient management. Addictive Disorders \& Their Treatment, 9(4), 143-149. [DOI:10.1097/ ADT.0b013e3181cf58f2]

Callaghan, R. C., Cunningham, J. K., Allebeck, P., Arenovich, T., Sajeev, G., Remington, G., et al. (2012). Methamphetamine use and schizophrenia: A population-based cohort study in California. American Journal of Psychiatry, 169(4), 389-396. [DOI:10.1176/appi.ajp.2011.10070937] [PMID]

Chen, C. K., Lin, S. K., Sham, P. C., Ball, D., Loh, E. W., \& Murray, R. M. (2005). Morbid risk for psychiatric disorder among the relatives of methamphetamine users with and without psychosis. American Journal of Medical Genetics Part B: Neuropsychiatric Genetics, 136B(1), 87-91. [DOI:10.1002/ ajmg.b.30187] [PMID]

Eghtedari, A., Shariat, V., \& Farahani, H. (2012). [The comparison of cognitive functions in patients with methamphetamine induced psychosis and control group (Persian)]. Ad- 
vances in Cognitive Sciences, 13(4), 19-26. https://icssjournal. ir/article-1-390-en.html

Ezzatpanah, Z., Shariat, S. V., \& Tehrani-Doost, M. (2014). Cognitive functions in methamphetamine induced psychosis compared to schizophrenia and normal subjects. Iranian Journal of Psychiatry, 9(3), 152-157. [PMCID]

Hajebi, A., Amini, H., Kashani, L., \& Sharifi, V. (2018). Twelvemonth course and outcome of methamphetamine-induced psychosis compared with first episode primary psychotic disorders. Early Intervention in Psychiatry, 12(5), 928-934. [DOI:10.1111/eip.12404] [PMID]

Hall, R. C. (1995). Global assessment of functioning: A modified scale. Psychosomatics, 36(3), 267-275. [DOI:10.1016/ S0033-3182(95)71666-8]

Hides, L., Dawe, S., McKetin, R., Kavanagh, D. J., Young, R. M. Teesson, M., et al. (2015). Primary and substance-induced psychotic disorders in methamphetamine users. Psychiatry Research, 226(1), 91-96. [DOI:10.1016/j.psychres.2014.11.077] [PMID]

Ikeda, M., Okahisa, Y., Aleksic, B., Won, M., Kondo, N., Naruse, N., et al. (2013). Evidence for shared genetic risk between methamphetamine-induced psychosis and schizophrenia. Neuropsychopharmacology, 38(10), 1864-1870. [DOI:10.1038/ npp.2013.94] [PMID] [PMCID]

Kay, S. R., Fiszbein, A., \& Opler, L. A. (1987). The Positive And Negative Syndrome Scale (PANSS) for schizophrenia. Schizophrenia Bulletin, 13(2), 261-276. [DOI:10.1093/schbul/13.2.261] [PMID]

Li, H., Lu, Q., Xiao, E., Li, Q., He, Z., \& Mei, X. (2014). Methamphetamine enhances the development of schizophrenia in firstdegree relatives of patients with schizophrenia. The Canadian Journal of Psychiatry, 59(2), 107-113. [DOI:10.1177/07067437140 5900206] [PMID] [PMCID]

Lohr, K. M., Stout, K. A., Dunn, A. R., Wang, M., Salahpour, A., Guillot, T. S., et al. (2015). Increased vesicular monoamine transporter 2 (VMAT2; Slc18a2) protects against methamphetamine toxicity. ACS Chemical Neuroscience, 6(5), 790-799. [DOI:10.1021/acschemneuro.5b00010] [PMID] [PMCID]

Medhus, S., Mordal, J., Holm, B., Mørland, J., \& Bramness, J. G. (2013). A comparison of symptoms and drug use between patients with methamphetamine associated psychoses and patients diagnosed with schizophrenia in two acute psychiatric wards. Psychiatry Research, 206(1), 17-21. [DOI:10.1016/j. psychres.2012.09.023] [PMID]

Okada, N., Takahashi, K., Nishimura, Y., Koike, S., Ishii-Takahashi, A., Sakakibara, E., et al. (2016). Characterizing prefrontal cortical activity during inhibition task in methamphetamine-associated psychosis versus schizophrenia: A multi-channel near-infrared spectroscopy study. Addiction Biology, 21(2), 489-503. [DOI:10.1111/adb.12224] [PMID]

Overall, J. E., \& Gorham, D. R. (1962). The brief psychiatric rating scale. Psychological Reports, 10(3), 799-812. [DOI:10.2466/ pr0.1962.10.3.799]

Samii, A. W. (2003). Drug abuse: Iran's" Thorniest Problem". The Brown Journal of World Affairs, 9(2), 283-299. https:/ / www.jstor.org/stable/24590484

Shin, E. J., Duong, C. X., Nguyen, X. K. T., Li, Z., Bing, G., Bach, J. H., et al. (2012). Role of oxidative stress in methamphetamine-induced dopaminergic toxicity mediated by protein kinase Cס. Behavioural Brain Research, 232(1), 98-113. [DOI:10.1016/j.bbr.2012.04.001] [PMID] [PMCID]

Srisurapanont, M., Ali, R., Marsden, J., Sunga, A., Wada, K., \& Monteiro, M. (2003). Psychotic symptoms in methamphetamine psychotic in-patients. International Journal of Neuropsychopharmacology, 6(4), 347-352. [DOI:10.1017/ S1461145703003675] [PMID]

Srisurapanont, M., Arunpongpaisal, S., Wada, K., Marsden, J., Ali, R., \& Kongsakon, R. (2011). Comparisons of methamphetamine psychotic and schizophrenic symptoms: A differential item functioning analysis. Progress in NeuroPsychopharmacology and Biological Psychiatry, 35(4), 959-964. [DOI:10.1016/j.pnpbp.2011.01.014] [PMID]

Ujike, H., \& Sato, M. (2004). Clinical features of sensitization to methamphetamine observed in patients with methamphetamine dependence and psychosis. Annals of the New York Academy of Sciences, 1025(1), 279-287. [DOI:10.1196/annals.1316.035] [PMID]

Vidal-Infer, A., Roger-Sánchez, C., Daza-Losada, M., Aguilar, M. A., Miñarro, J., \& Rodríguez-Arias, M. (2012). Role of the dopaminergic system in the acquisition, expression and reinstatement of MDMA-induced conditioned place preference in adolescent mice. Plos One, 7(8), e43107. [DOI:10.1371/journal.pone.0043107] [PMID] [PMCID]

Wearne, T. A., \& Cornish, J. L. (2018). A comparison of methamphetamine-induced psychosis and schizophrenia: A review of positive, negative, and cognitive symptomatology. Frontiers in Psychiatry, 9, 491. [DOI:10.3389/fpsyt.2018.00491] [PMID] [PMCID]

Zarghami, M. (2011). Methamphetamine has changed the profile of patients utilizing psychiatric emergency services in Iran. Iranian Journal of Psychiatry and Behavioral Sciences, 5(1), 1-5. https:// brieflands.com/articles/ijpbs-2924.html 
This Page Intentionally Left Blank 